\title{
Computed Tomography-Estimated Pancreatic Steatosis is a Risk Factor for Carotid Plaque in Type 2 Diabetes Mellitus Patients: A Cross-Sectional Study
}

\section{Pengtao Sun}

Beijing Shijitan Hospital

Chunzhi Fan

Beijing ChaoYang Hospital

Rengui Wang

Beijing Shijitan Hospital

Tongwei Chu

Beijing Shijitan Hospital

\section{Xiaoli Sun}

Beijing Shijitan Hospital

Dongxue Zhang

Beijing Shijitan Hospital

Xuechao Du ( $\nabla$ duxuechao@ccmu.edu.cn )

Beijing Chaoyang Hospital https://orcid.org/0000-0002-5491-0398

Original investigation

Keywords: Pancreas steatosis, Computed tomography, Diabetes mellitus, Atherosclerosis

Posted Date: December 17th, 2020

DOI: https://doi.org/10.21203/rs.3.rs-127501/v1

License: (c) (1) This work is licensed under a Creative Commons Attribution 4.0 International License. Read Full License 


\section{Abstract}

Background Pancreatic steatosis correlates with the thickness of arterial intima. However, the correlation between pancreatic steatosis and carotid atherosclerosis plaque, which better predict the prognosis of cardiovascular disease, is unclear. We aimed to explore potential effects of pancreatic fat content measured by computer tomography (CT) on carotid plaque in patients with type 2 diabetes mellitus (T2DM).

Methods Patients with T2DM who underwent CT scan of the upper abdomen and ultrasound of the carotid artery were consecutively enrolled. Based on ultrasound results, the patients were divided into non-plaque group and plaque group, and the latter was categorized into hypoechoic plaque subgroup and non-hypoechoic plaque subgroup. The CT attenuation of pancreas and spleen were measured. Pancreasto-spleen attenuation ratio (P/S) and difference between pancreatic and splenic attenuation (P-S) were calculated. The cut-off values of $\mathrm{P} / \mathrm{S}$ and $\mathrm{P}-\mathrm{S}$ were obtained using receiver operating characteristic (ROC) curves. Logistic regression models were used to evaluate association of $\mathrm{P} / \mathrm{S}$ or $\mathrm{P}-\mathrm{S}$ with carotid plaque or hypoechoic plaque.

Results A total of 337 patients with T2DM were enrolled, including 101 cases (30.0\%) in the non-plaque group, 146 cases (43.3\%) in the hypoechoic plaque subgroup, and 90 cases $(26.7 \%)$ in the nonhypoechoic plaque subgroup. P/S and P-S in plaque group were lower than those in non-plaque group, with a cut-off value of P/S and P-S as 0.72 and -13.33 , respectively. After adjusting for risk factors, $\mathrm{P} / \mathrm{S}$ and P-S correlated with carotid plaque [for low P/S: OR (95\% Cl): 3.15 (1.47-6.73), P=0.0031; for low P-S: OR (95\% Cl): 2.84 (1.42-5.66), $\mathrm{P}=0.0031$ ] as well as carotid hypoechoic plaque [for low $\mathrm{P} / \mathrm{S}$ : OR (95\% Cl): 1.82 (1.07-3.08), $P=0.0259$; for low P-S: OR (95\% Cl): 1.82 (1.09-3.02), $P=0.021]$.

Conclusions T2DM patients with carotid plaque have higher pancreatic fat content than those without. Pancreatic steatosis correlates with carotid plaque and hypoechoic plaque in T2DM patients.

\section{Background}

Carotid plaque is the main pathological feature of carotid atherosclerosis, which is closely related to the development of cerebral infarction [1]. Furthermore, hypoechoic carotid plaque associated with adverse cerebrovascular outcome independent of stenosis severity [2]. To evaluate risk factors of carotid plaque for cerebral infarction will facilitate its prevention and treatment. Ectopic fat deposition, characterized by accumulation of fat tissue in the liver, pancreas, and skeletal muscle [3], may release various bioactive factors to promote the development of cardiovascular diseases [4]. Ectopic fat deposition in the pancreas is defined as pancreatic steatosis [5]. Histology is the gold standard for diagnosis of pancreatic steatosis. However, it is an invasive examination, which limits its wide application. Computed tomography (CT) quantitative measurement of pancreatic fat content has high accuracy, proven to be a reliable noninvasive method [6]. In recent years, pancreatic steatosis was diagnosed by CT in burgeoning literatures $[7,8]$. 
Although several reports indicated relationship between pancreatic steatosis measured by CT and atherosclerosis, most of them focused on arterial intima-media or intima thickness $[9,10]$. However, carotid plaque may provide more direct evidence of subclinical atherosclerosis than intimal thickness. In addition, predictive value of carotid plaque for cardiovascular adverse events may be higher than carotid intima-media thickness [11]. Thus, demonstrating relationship between pancreatic steatosis and carotid plaque may be more useful to identify patients at high risk of ischemic stroke. Indeed, pancreatic steatosis was related to arterial calcified plaque in healthy people [12]. However, there are very few reports on the relationship between pancreatic steatosis and carotid plaque (especially hypoechoic plaque) in patients with type 2 diabetes mellitus (T2DM). Therefore, our study aims to investigate if pancreatic steatosis confers increased risk of carotid plaque in patients with T2DM.

\section{Methods}

This study retrospectively analyzed 337 patients with T2DM from January 2016 to January 2020. Inclusion criteria were as follows: (1) age $\geq 20$ years old; (2) diagnosis according to 2010 American Diabetes Association standards [13]; (3) with complete information on upper abdominal CT scan, carotid artery ultrasound and biochemical examination; and (4) all the above examinations were performed within 2 weeks. Exclusion criteria were as follows: (1) complicated pancreatic diseases (e.g., acute or chronic pancreatitis, pancreatic neoplasms); (2) liver and kidney failure; (3) systemic infectious diseases; (4) malignant tumors; (5) treatment with glucocorticoids. Basic information was recorded, including age, gender, blood pressure, height, weight, and smoking history, diabetes duration and biochemical examination.

\section{Carotid Ultrasound Examination And Plaque Assessment}

An ultrasonic detector (Philips iE33 or GE Logic E9) with a $10 \mathrm{MHz}$ linear array probe was used to evaluate carotid artery. Patients were examined in supine position, and intima-media thickness (IMT) of bilateral carotid arteries (including common and internal carotid artery, as well as bifurcations) were measured. Carotid plaque was defined as IMT $\geq 1.5 \mathrm{~mm}$ or local thickness exceeding $50 \%$ of the adjacent lumen [14]. Plaques were classified into hypoechoic plaque and non-hypoechoic (either mixed echoic or hyperechoic) plaque. According to the presence or absence of carotid plaque, patients were divided into non-plaque group and plaque group. The plaque group were classified into hypoechoic plaque subgroup and non-hypoechoic plaque subgroup according to the presence or absence of hypoechoic plaque.

\section{Ct Scan And Pancreatic Fat Content Measurement}

Upper abdominal CT scan was performed on a spiral CT scanner (Siemens Somatom Sensation 16 CT or Brilliance iCT, Philips Healthcare). Scan parameters were as follows: kVp, 100-120; mAs, 100-250; pitch 1; slice thickness and slice spacing, $2 \mathrm{~mm}$. Attenuation within the head, body and tail, respectively, of the pancreas was measured on CT axial images, where the largest diameter of anatomical part was 
observed. The ROI area was $1.0 \mathrm{~cm}^{2}$. The main pancreatic duct, pancreatic edge and splenic vessels were carefully avoided during measurement. CT attenuation of 3 different parts of the spleen was measured. The average CT attenuation of the pancreas and spleen, respectively, was calculated. The difference between pancreatic and splenic attenuation (P-S), and pancreas-to-spleen attenuation ratio (P/S) were calculated.

\section{Statistical analysis}

Statistical analysis was performed using SAS version 9.1 (SAS, Cary, NC, USA). Basic clinical data and CT measurements were compared by using Kruskal-Wallis rank test or chi-square test. Cutoff values of $P / S$ and P-S were obtained using receiver operating characteristic (ROC) curves. Low P/S or low P-S was defined as pancreatic steatosis. Carotid plaque or hypoechoic plaque was considered as dependent variable. Univariate logistic regression was created to analyze relationship of plaque with P/S or P-S. Statistically significant factors in univariate logistic regression as well as clinical factors, such as age, gender, obesity (body mass index $\geq 30 \mathrm{~kg} / \mathrm{m}^{2}$ ), duration of diabetes, hypertension, smoking, total cholesterol, triglycerides and high-density lipoprotein, were selected for multivariate logistic regression. Statistical significance was defined at a $p<0.05$ (2-sided).

\section{Results}

\section{Patients characteristics}

A total of 337 patients with T2DM were enrolled in this study, with an average age of $60.1 \pm 11.7$ years old. Among them, 201 cases (59.6\%) were male with an average age of $57.4 \pm 11.9$ years old, while 136 cases $(40.4 \%)$ were female with an average age of $64.2 \pm 10.3$ years old. There were 101 cases $(30.0 \%)$ in non-plaque group, whereas 236 cases $(70.0 \%)$ in plaque group; 146 cases in hypoechoic plaque subgroup (43.3\%) and 90 cases (26.7\%) in non-hypoechoic plaque subgroup. Basic information of study population is summarized in Table 1. 
Table 1

Clinical and laboratory characteristics of study population

\begin{tabular}{|c|c|c|c|c|}
\hline \multirow[t]{2}{*}{ Index } & \multirow[t]{2}{*}{ Non-plaque } & \multicolumn{2}{|l|}{ Plaque } & \multirow[t]{2}{*}{ P-value } \\
\hline & & Hypoechoic & Non-hypoechoic & \\
\hline Male, n (\%) & $58(57.4)$ & $92(63.0)$ & $51(56.7)$ & 0.54 \\
\hline Age (years) & $52.8 \pm 11.8$ & $63.0 \pm 10.8$ & $63.7 \pm 9.2$ & $<.0001$ \\
\hline Smoking, n (\%) & $34(33.7)$ & $48(32.9)$ & $25(27.8)$ & 0.63 \\
\hline Hypertension, n (\%) & $55(54.4)$ & $108(73.9)$ & 75 (83.3) & $<.0001$ \\
\hline SBP (mm Hg) & $132.0 \pm 17.0$ & $134.8 \pm 19.1$ & $133.8 \pm 13.9$ & 0.45 \\
\hline $\mathrm{DBP}(\mathrm{mm} \mathrm{Hg})$ & $83.1 \pm 11.9$ & $81.2 \pm 11.0$ & $79.9 \pm 9.1$ & 0.38 \\
\hline MAP $(\mathrm{mm} \mathrm{Hg})$ & $99.4 \pm 12.6$ & $99.0 \pm 12.4$ & $97.8 \pm 9.2$ & 0.98 \\
\hline $\mathrm{BMI}\left(\mathrm{kg} / \mathrm{m}^{2}\right)$ & $26.1 \pm 4.1$ & $25.7 \pm 3.1$ & $26.0 \pm 3.2$ & 0.70 \\
\hline DD, IQR (years) & $6(1-10)$ & $10(5-16)$ & $14(6-20)$ & $<.0001$ \\
\hline $\mathrm{FPG}(\mathrm{mmol} / \mathrm{L})$ & $8.38 \pm 2.90$ & $8.67 \pm 4.03$ & $8.55 \pm 3.67$ & 0.92 \\
\hline HbA1c (\%) & $7.67 \pm 1.36$ & $8.05 \pm 2.14$ & $7.59 \pm 1.53$ & 0.80 \\
\hline $\mathrm{TC}(\mathrm{mmol} / \mathrm{L})$ & $4.70 \pm 1.24$ & $4.41 \pm 1.13$ & $4.16 \pm 0.96$ & 0.0074 \\
\hline TG, IQR (mmol/L) & $1.81(1.17-3.09)$ & $1.63(1.16-2.44)$ & $1.65(1.07-2.59)$ & 0.50 \\
\hline $\mathrm{HDL}-\mathrm{C}(\mathrm{mmol} / \mathrm{L})$ & $1.06 \pm 0.29$ & $0.99 \pm 0.25$ & $1.05 \pm 0.26$ & 0.09 \\
\hline LDL-C (mmol/L) & $2.41 \pm 0.83$ & $2.43 \pm 0.81$ & $2.26 \pm 0.80$ & 0.21 \\
\hline \multicolumn{5}{|c|}{$\begin{array}{l}S B P \text { Systolic blood pressure, } D B P \text { Diastolic blood pressure, Map Mean arterial pressure, } B M I \text { Body } \\
\text { mass index, } D D \text { Diabetes duration, } I Q R \text { interquartile range, } F P G \text { Fasting plasma glucose, } T C \text { Total } \\
\text { cholesterol, } T G \text { Triglycerides, } H D L-C \text { High density lipoprotein cholesterol, } L D L-C \text { Low density } \\
\text { lipoprotein cholesterol }\end{array}$} \\
\hline \multicolumn{5}{|c|}{$\begin{array}{l}\text { Patients in plaque group were elder, with longer duration of T2DM. Hypertension was more common } \\
\text { in plaque group. Total cholesterol level was higher in non-plaque group. Gender, smoking ratio, } \\
\text { systolic blood pressure, diastolic blood pressure, mean arterial pressure, BMI, fasting blood glucose, } \\
\text { HbA1c, triacylglycerol, high-density lipoprotein, and low-density lipoprotein were comparable between } \\
\text { the two groups. }\end{array}$} \\
\hline
\end{tabular}

\section{Ct Evaluation Of Pancreatic Steatosis}

Compared with non-plaque group, CT attenuation of the pancreas, P/S and P-S were lower in plaque group. There was no difference in CT attenuation of the spleen between the two groups (Table 2). 
Table 2

Comparison of pancreatic fat content

\begin{tabular}{|lllll|}
\hline Index & Non-plaque & \multicolumn{2}{l}{ Plaque } & P-value \\
\cline { 3 - 4 } & & Hypoechoic & Non-hypoechoic & \\
\hline Pancreas (HU) & $39.84 \pm 14.59$ & $35.42 \pm 13.35$ & $35.91 \pm 13.09$ & 0.001 \\
\hline Spleen $(\mathrm{HU})$ & $51.57 \pm 2.83$ & $51.16 \pm 3.15$ & $50.80 \pm 3.68$ & 0.29 \\
\hline P/S & $0.77 \pm 0.27$ & $0.69 \pm 0.25$ & $0.70 \pm 0.25$ & 0.0029 \\
\hline P-S & $-11.72 \pm 14.07$ & $-15.74 \pm 13.01$ & $-14.89 \pm 12.86$ & 0.004 \\
\hline P/SPancreas-to-spleen attenuation ratio & & \\
\hline
\end{tabular}

$P$-SDifference between pancreatic and splenic attenuation

Based on ROC curves, the cutoff value of P/S was 0.72 , and the area under the curve was 0.61 . The sensitivity and specificity of $\mathrm{P} / \mathrm{S}$ for predicting carotid plaque was $41.1 \%$ and $80.2 \%$, respectively $(\mathrm{P}=$ 0.0005 ). The cutoff value of P-S was -13.33 , and the area under the curve was 0.61 . The sensitivity and specificity of P-S for predicting carotid plaque was $45.8 \%$ and $75.3 \%$, respectively $(P=0.0009)$. Patients with low P/S ( $\leq 0.72)$ or low P-S $(\leq-13.33)$ was defined as pancreatic steatosis. Patients with low P/S or low P-S accounted for $34.7 \%(n=117)$ and $39.5 \%(n=133)$, respectively.

\section{Risk Factors For Carotid Plaque}

As shown in Table 3, univariate logistic regression model identified low $\mathrm{P} / \mathrm{S}(\mathrm{OR}=2.83,95 \% \mathrm{Cl}=1.62-$ $4.91, \mathrm{P}=0.0002)$ and low $\mathrm{P}-\mathrm{S}(\mathrm{OR}=2.57,95 \% \mathrm{Cl}=1.52-4.31, \mathrm{P}=0.0004)$ as risk factors of carotid plaque. Furthermore, low $\mathrm{P} / \mathrm{S}(\mathrm{OR}=1.93,95 \% \mathrm{Cl}=1.22-3.03, \mathrm{P}=0.0047)$ and low $\mathrm{P}-\mathrm{S}(\mathrm{OR}=1.97,95 \% \mathrm{Cl}$ $=1.26-3.07, P=0.0028)$ were risk factors of hypoechoic plaque. 
Table 3

Univariate logistic regression models for carotid plaque and hypoechoic plaque

\begin{tabular}{|c|c|c|c|c|}
\hline \multirow[t]{2}{*}{ Factor } & \multicolumn{2}{|l|}{ Plaque } & \multicolumn{2}{|l|}{ Hypoechoic plaque } \\
\hline & OR $(95 \% \mathrm{Cl})$ & P-value & OR $(95 \% \mathrm{Cl})$ & P-value \\
\hline Low P/S & $2.83(1.62-4.91)$ & 0.0002 & $1.93(1.22-3.03)$ & 0.0047 \\
\hline Low P-S & $2.57(1.52-4.31)$ & 0.0004 & $1.97(1.26-3.07)$ & 0.0028 \\
\hline Age ( $\geq 60$ years) & $4.78(2.85-7.99)$ & $<.0001$ & $2.24(1.43-3.47)$ & 0.0004 \\
\hline Male & $1.14(0.71-1.82)$ & 0.59 & $1.28(0.82-1.99)$ & 0.27 \\
\hline Smoking & $0.88(0.53-1.45)$ & 0.62 & $1.10(0.69-1.74)$ & 0.70 \\
\hline Obesity & $0.58(0.27-1.18)$ & 0.13 & $0.65(0.31-1.35)$ & 0.25 \\
\hline Hypertension & $2.89(1.75-4.74)$ & $<.0001$ & $1.33(0.82-2.15)$ & 0.24 \\
\hline SBP & $1.01(0.99-1.02)$ & 0.25 & $1.01(0.99-1.01)$ & 0.31 \\
\hline DBP & $0.98(0.95-1.01)$ & 0.06 & $0.99(0.97-1.01)$ & 0.72 \\
\hline MAP & $0.99(0.97-1.01)$ & 0.54 & $1.00(0.98-1.02)$ & 0.78 \\
\hline DD & $1.10(1.05-1.13)$ & $<.0001$ & $1.01(0.98-1.04)$ & 0.34 \\
\hline FPG & $1.02(0.95-1.09)$ & 0.57 & $1.02(0.95-1.08)$ & 0.59 \\
\hline $\mathrm{HbA1c}$ & $1.07(0.85-1.33)$ & 0.55 & $1.14(0.93-1.38)$ & 0.20 \\
\hline TC & $0.74(0.60-0.92)$ & 0.0069 & $0.97(0.79-1.17)$ & 0.74 \\
\hline TG & $0.89(0.81-0.98)$ & 0.0207 & $0.97(0.88-1.06)$ & 0.52 \\
\hline HDL-C & $0.51(0.21-1.20)$ & 0.13 & $0.40(0.17-0.93)$ & 0.0346 \\
\hline LDL-C & $0.94(0.69-1.25)$ & 0.66 & $1.15(0.87-1.50)$ & 0.32 \\
\hline \multicolumn{5}{|c|}{$\begin{array}{l}P / S \text { Pancreas-to-spleen attenuation ratio, } P \text {-S Difference between pancreatic and splenic attenuation, } \\
S B P \text { systolic blood pressure, } D B P \text { Diastolic blood pressure, Map Mean arterial pressure, } D D \text { Diabetes } \\
\text { duration, } F P G \text { Fasting plasma glucose, } H b A 1 c \text { glycated hemoglobin, } T C \text { Total cholesterol, } T G \\
\text { Triglycerides, } H D L-C \text { High density lipoprotein cholesterol, } L D L-C \text { Low density lipoprotein cholesterol }\end{array}$} \\
\hline
\end{tabular}

After adjusting for conventional cardiovascular risk factors (including age, gender, smoking, duration of diabetes, hypertension, obesity, total cholesterol, triglycerides, high-density lipoprotein), multivariate logistic regression model identified low $\mathrm{P} / \mathrm{S}(\mathrm{OR}=3.15,95 \% \mathrm{Cl}=1.47-6.73, \mathrm{P}=0.0031)$ and low $\mathrm{P}-\mathrm{S}(\mathrm{OR}$ $=2.84,95 \% \mathrm{Cl}=1.42-5.66, \mathrm{P}=0.0031$ ) as independent risk factors of carotid plaque (Table 4).

Furthermore, low $\mathrm{P} / \mathrm{S}(\mathrm{OR}=1.82,95 \% \mathrm{Cl}=1.07-3.08, \mathrm{P}=0.0259)$ and low $\mathrm{P}-\mathrm{S}(\mathrm{OR}=1.82,95 \% \mathrm{Cl}=1.09$ $3.02, \mathrm{P}=0.021$ ) were independent risk factors of hypoechoic plaque (Table 5 ). 
Table 4

Multivariate logistic regression models for carotid plaque

\begin{tabular}{|lllll|}
\hline Factor & Low P/S & & Low P-S & \\
\cline { 2 - 5 } & OR $(95 \%$ Cl) & P-value & OR $(95 \%$ Cl) & P-value \\
\hline Low P/S & $3.15(1.47-6.73)$ & 0.0031 & - & - \\
\hline Low P-S & - & - & $2.84(1.42-5.66)$ & 0.0031 \\
\hline Age ( $\geq 60$ years) & $4.56(2.20-9.44)$ & $<.0001$ & $4.68(2.25-9.69)$ & $<.0001$ \\
\hline Male & $2.34(1.03-5.29)$ & 0.041 & $2.39(1.06-5.39)$ & 0.0354 \\
\hline Smoking & $0.98(0.45-2.11)$ & 0.97 & $1.02(0.47-2.18)$ & 0.97 \\
\hline Obesity & $1.03(0.41-2.55)$ & 0.95 & $0.99(0.40-2.45)$ & 0.99 \\
\hline Hypertension & $2.73(1.42-5.20)$ & 0.0024 & $2.96(1.55-5.62)$ & 0.001 \\
\hline DD & $1.08(1.02-1.13)$ & 0.0021 & $1.08(1.02-1.13)$ & 0.0022 \\
\hline TC & $1.07(0.76-1.51)$ & 0.68 & $1.04(0.73-1.46)$ & 0.84 \\
\hline TG & $0.89(0.74-1.05)$ & 0.17 & $0.91(0.76-1.07)$ & 0.25 \\
\hline HDL-C & $0.33(0.07-1.34)$ & 0.12 & $0.38(0.09-1.53)$ & 0.17 \\
\hline
\end{tabular}

$P / S$ Pancreas-to-spleen attenuation ratio, $P$-S Difference between pancreatic and splenic attenuation, $T C$ Total cholesterol, TG Triglycerides, HDL-CHigh density lipoprotein cholesterol 
Table 5

Multivariate logistic regression models for carotid hypoechoic plaque

\begin{tabular}{|lllll|}
\hline Factor & \multicolumn{2}{l}{ Low P/S } & & Low P-S \\
\cline { 2 - 5 } & OR (95\% Cl) & P-value & OR (95\% Cl) & P-value \\
\hline Low P/S & $1.82(1.07-3.08)$ & 0.0259 & - & - \\
\hline Low P-S & - & - & $1.82(1.09-3.02)$ & 0.021 \\
\hline Age ( $\geq 60$ years) & $2.05(1.14-3.65)$ & 0.0151 & $2.05(1.15-3.66)$ & 0.0148 \\
\hline Male & $1.26(0.68-2.32)$ & 0.46 & $1.27(0.68-2.33)$ & 0.45 \\
\hline Smoking & $1.24(0.67-2.28)$ & 0.48 & $1.27(0.68-2.34)$ & 0.44 \\
\hline Obesity & $0.80(0.35-1.80)$ & 0.60 & $0.78(0.34-1.76)$ & 0.56 \\
\hline Hypertension & $1.20(0.69-2.07)$ & 0.51 & $1.23(0.71-2.12)$ & 0.45 \\
\hline DD & $1.00(0.96-1.03)$ & 0.97 & $1.00(0.96-1.03)$ & 0.96 \\
\hline TC & $1.26(0.94-1.67)$ & 0.11 & $1.24(0.93-1.64)$ & 0.14 \\
\hline TG & $0.92(0.79-1.05)$ & 0.23 & $0.93(0.80-1.06)$ & 0.28 \\
\hline HDL-C & $0.27(0.08-0.87)$ & 0.0286 & $0.29(0.08-0.93)$ & 0.0377 \\
\hline
\end{tabular}

$P / S$ Pancreas-to-spleen attenuation ratio, $P$-S Difference between pancreatic and splenic attenuation, $T C$ Total cholesterol, TG Triglycerides, HDL-C High density lipoprotein cholesterol

\section{Discussion}

Our study has demonstrated that P/S and P-S in T2DM patients with carotid plaque, especially with hypoechoic plaque, are lower than those without carotid plaque. After adjusting for covariates, multivariate logistic regression model identifies low P/S and low P-S as independent risk factors for carotid plaque, in particular carotid hypoechoic plaque.

\section{Ct Quantitative Evaluation Of Pancreatic Steatosis}

Pancreatic steatosis has been indicated in various diseases $[5,7,8]$. Therefore, a simple and accurate method for evaluating pancreatic fat content is of clinical significance. Ultrasound and magnetic resonance imaging (MRI) have been used to measure pancreatic fat content $[15,16]$. However, ultrasound results may be affected by the operator's experience, while MRI is expensive and time-consuming, which limit their clinical application. Histologically, pancreatic CT density index (P/S, P-S) had a good correlation with pancreatic fat content [6]. Therefore, CT, which is widely used in clinical practice, is a 
reliable tool to evaluate pancreatic fat content. Rather than CT attenuation, P/S and P-S were applied to quantify pancreatic fat content in many studies [17-19], which was also applied in this study.

At present, no uniform CT cutoff value has been applied for the diagnosis of pancreatic steatosis. In this study, cutoff values of P/S (0.72) and P-S (-13.33) were obtained from ROC curves, consistent with previous reports $[5,20]$. In this study, there were 117 patients $(34.7 \%)$ with low $\mathrm{P} / \mathrm{S}$ and 133 patients (39.5\%) with low PS. The incidence of pancreatic steatosis was higher than previously reported in healthy Chinese adults [21], indicating that diabetes patients are more likely to suffer from pancreatic steatosis [22].

\section{Relationship Between Pancreatic Steatosis And Carotid Plaque}

Ectopic fat deposition is closely related to atherosclerotic plaque and cardiovascular disease [23]. Pancreatic steatosis is manifestation of ectopic fat deposition, characterized by fat accumulation in the pancreas [5, 24]. Pancreatic steatosis was related to carotid-femoral pulse wave velocity, which might predict carotid atherosclerosis in patients with non-alcoholic fatty liver disease [9]. Pancreatic steatosis was in proportion to aortic intima thickness [25] and systemic arterial calcification [12]. Ectopic fat deposition in the pancreas increased risk for carotid atherosclerosis in non-obese T2DM patients, but not in obese patients [26]. In this study, after adjusting for obesity and other traditional risk factors, low P/S and low P-S remain correlated with carotid plaque. Thus, pancreatic steatosis is an independent risk factor for carotid atherosclerosis.

Hypoechoic plaque is characteristic manifestation of unstable plaque, which is highly enriched in lipids and more prone to cerebrovascular diseases [27]. Previous study reported that hypoechoic plaque was independently related to progression of atherosclerosis [28]. The size of juxtaluminal hypoechoic area in asymptomatic carotid plaque linearly correlated with the risk of stroke [29]. Additionally, carotid hypoechoic plaque predicted adverse cardiovascular events in asymptomatic carotid stenosis [30]. Furthermore, hypoechoic plaque indicated cognitive impairment among patients with acute ischemic stroke [31]. We propose that pancreatic steatosis confers increased risk of hypoechoic carotid plaque. Therefore, for patients with T2DM, pancreatic fat content on CT images can help identify those at high risk of stroke, and guide early clinical intervention to prevent cerebrovascular diseases.

Pathological mechanisms underlying how pancreatic steatosis increases susceptibility to carotid plaque remain unclear. Pancreatic steatosis impairs insulin secretion in patients with T2DM [32], resulting in decreased insulin secretion. Moreover, pancreatic steatosis may contribute to insulin resistance [33], leading to relatively insufficient insulin. Consequently, elevated blood glucose causes damage to blood vessel wall through a variety of molecular pathways [34], and ultimately promotes the formation of atherosclerosis plaque. 
There are several limitations in the current study. Firstly, ultrasound, instead of MRI, is applied in this study. Although MRI is superior to ultrasound in evaluating plaque composition, it is not widely used in clinical practice, since most radiologists have insufficient experience in this field. By contrast, ultrasound is easy to operate and widely applied to evaluate carotid plaque. Although ultrasound is not accurate enough to distinguish stable or unstable plaque according to echo features, hypoechoic plaque remains a certain value in predicting cardiovascular events $[29,30]$. Secondly, all subjects in this study are patients with T2DM and cannot represent the general population. Thirdly, as a cross-sectional study, cause and effect relationship between pancreatic steatosis and carotid plaque needs to be further explored in future prospective studies with larger sample sizes.

\section{Conclusions}

In conclusion, T2DM patients with carotid plaque, especially hypoechoic plaque, may have more pancreatic fat content. Pancreatic steatosis may confer an independent increased risk of carotid (hypoechoic) plaque in T2DM patients.

\section{Abbreviations}

BMI: body mass index; CT: computer tomography; DBP: diastolic blood pressure; DD: diabetes duration; IQR: interquartile range; FPG: fasting plasma glucose; HbA1c: glycated hemoglobin; HDL-C: high density lipoprotein cholesterol; IMT: intima-media thickness; LDL-C: low density lipoprotein cholesterol; Map: mean arterial pressure; MRI: magnetic resonance imaging; P/S: pancreas-to-spleen attenuation ratio; P-S: the difference between pancreatic and splenic attenuation; SBP: systolic blood pressure; TC: total cholesterol; TG: triglycerides; T2DM: type 2 diabetes mellitus.

\section{Declarations}

\section{Ethics approval and consent to participate}

This study has been approved by Ethics Committee of Institutional Review Board (IRB) at Beijing Shijitan Hospital (sjtkyll-Ix-2020 (36)).

\section{Consent for publication}

Not applicable.

\section{Availability of data and materials}

All data generated or analyzed during this study are included in this published article.

\section{Competing interests}


All the authors declare that they have no conflict of interest.

\section{Funding}

Not applicable.

\section{Authors' contributions}

All authors contributed to the content and critical review of the manuscript. All authors read and approved the final manuscript.

\section{Acknowledgements}

Not applicable.

\section{References}

1. Zhou D, Li J, Liu D, Ji L, Wang N, Deng J, et al. Irregular surface of carotid atherosclerotic plaque is associated with ischemic stroke: a magnetic resonance imaging study. J Geriatr Cardiol. 2019;16:872-9.

2. Jashari F, Ibrahimi P, Bajraktari G, Grönlund C, Wester P, Henein MY. Carotid plaque echogenicity predicts cerebrovascular symptoms: a systematic review and meta-analysis. Eur J Neurol. 2016;23:1241-7.

3. Pieńkowska J, Brzeska B, Kaszubowski M, Kozak O, Jankowska A, Szurowska E. The correlation between the MRI-evaluated ectopic fat accumulation and the incidence of diabetes mellitus and hypertension depends on body mass index and waist circumference ratio. PLoS One. 2020;15:e226889.

4. Lim S, Meigs JB. Links between ectopic fat and vascular disease in humans. Arterioscler Thromb Vasc Biol. 2014;34:1820-6.

5. Dite P, Blaho M, Bojkova M, Jabandziev P, Kunovsky L. Nonalcoholic fatty pancreas disease: clinical consequences. Dig Dis. 2020;38:143-9.

6. Kim SY, Kim H, Cho JY, Lim S, Cha K, Lee KH, et al. Quantitative assessment of pancreatic fat by using unenhanced CT: pathologic correlation and clinical implications. Radiology. 2014;271:104-12.

7. Kashiwagi K, Seino T, Fukuhara S, Minami K, Horibe M, Iwasaki E, et al. Pancreatic fat content detected by computed tomography and its significant relationship with intraductal papillary mucinous neoplasm. Pancreas. 2018;47:1087-92.

8. Jeong JS, Kim MK, Han KD, Hong OK, Baek K, Song K, et al. The association between pancreatic steatosis and diabetic retinopathy in type 2 diabetes mellitus patients. Diabetes Metab J. 2018;42:425-32.

9. Ozturk K, Dogan T, Celikkanat S, Ozen A, Demirci H, Kurt O, et al. The association of fatty pancreas with subclinical atherosclerosis in nonalcoholic fatty liver disease. Eur J Gastroenterol Hepatol. 
2018;30:411-7.

10. Sotoudehmanesh R, Tahmasbi A, Sadeghi A, Hosseini H, Mohamadnejad M. The prevalence of nonalcoholic fatty pancreas by endoscopic ultrasonography. Pancreas. 2019;48:1220-4.

11. Inaba Y, Chen JA, Bergmann SR. Carotid plaque, compared with carotid intima-media thickness, more accurately predicts coronary artery disease events: a meta-analysis. Atherosclerosis. 2012;220:12833.

12. Koo BK, Denenberg JO, Wright CM, Criqui MH, Allison MA. The association between pancreatic fat and systemic calcified atherosclerosis. Pancreas. 2020;49:e16-8.

13. American Diabetes Association. Standards of medical care in diabetes-2010. Diabetes Care. 2010;33(Suppl 1):11-61.

14. Li M, Zhao C, Li T, Tu Y, Lu J, Zhang R, et al. The coexistence of carotid and lower extremity atherosclerosis further increases cardio-cerebrovascular risk in type 2 diabetes. Cardiovasc Diabetol. 2016;15:43.

15. Wang CY, Ou HY, Chen MF, Chang TC, Chang CJ. Enigmatic ectopic fat: prevalence of nonalcoholic fatty pancreas disease and its associated factors in a Chinese population. J Am Heart Assoc. 2014;3:e297.

16. Yao WJ, Guo Z, Wang L, Li K, Saba L, Guglielmi G, et al. Pancreas fat quantification with quantitative CT: an MRI correlation analysis. Clin Radiol. 2020;75:391-7.

17. Xie J, Xu L, Pan Y, Li P, Liu Y, Pan Y, et al. Nonalcoholic fatty pancreas disease is related independently to the severity of acute pancreatitis. Eur J Gastroenterol Hepatol. 2019;31:973-8.

18. Koc U, Taydas $\mathrm{O}$. Investigation of the relationship between fatty pancreas and cholecystectomy using noncontrast computed tomography. J Med Imaging Radiat Sci. 2019;50:220-6.

19. Ahbab S, Ünsal A, Ataoğlu HE, Can TS, Kayaş D, Savaş Y. Prediabetes and type 2 diabetes are independent risk factors for computed tomography-estimated nonalcoholic fatty pancreas disease. Clinics. 2019;74:e1337.

20. Fukuda Y, Yamada D, Eguchi H, Hata T, Iwagami Y, Noda T, et al. CT density in the pancreas is a promising imaging predictor for pancreatic ductal adenocarcinoma. Ann Surg Oncol. 2017;24:27629.

21. Zhou J, Li M, Zhang D, Lin H, Dai X, Sun X, et al. The correlation between pancreatic steatosis and metabolic syndrome in a Chinese population. Pancreatology. 2016;16:578-83.

22. Ou HY, Wang CY, Yang YC, Chen MF, Chang CJ. The association between nonalcoholic fatty pancreas disease and diabetes. PLoS One. 2013;8:e62561.

23. Ferrara D, Montecucco F, Dallegri F, Carbone F. Impact of different ectopic fat depots on cardiovascular and metabolic diseases. J Cell Physiol. 2019;234:21630-41.

24. Pezzilli R. Pancreatic steatosis: Is it related to either obesity or diabetes mellitus? World J Diabetes. 2014;5:415-9. 
25. Kul S, Karadeniz A, Dursun İ, Şahin S, Faruk ÇÖ, Raşit SM, et al. Non-alcoholic fatty pancreas disease is associated with increased epicardial adipose tissue and aortic intima-media thickness. Acta Cardiol Sin. 2019;35:118-25.

26. Kim MK, Chun HJ, Park JH, Yeo DM, Baek KH, Song KH, et al. The association between ectopic fat in the pancreas and subclinical atherosclerosis in type 2 diabetes. Diabetes Res Clin Pract. 2014;106:590-6.

27. Picano E, Paterni M. Ultrasound tissue characterization of vulnerable atherosclerotic plaque. Int $J$ Mol Sci. 2015;16:10121-33.

28. Liu Y, Hua Y, Liu R, Wang L, Duan C, Ling C, et al. Ultrasonographical features associated with progression of atherosclerosis in patients with moderate internal carotid artery stenosis. Transl Stroke Res. 2018;9:375-81.

29. Kakkos SK, Griffin MB, Nicolaides AN, Kyriacou E, Sabetai MM, Tegos T, et al. The size of juxtaluminal hypoechoic area in ultrasound images of asymptomatic carotid plaques predicts the occurrence of stroke. J Vasc Surg. 2013;57:609-18.

30. Gupta A, Kesavabhotla K, Baradaran H, Kamel H, Pandya A, Giambrone AE, et al. Plaque echolucency and stroke risk in asymptomatic carotid stenosis. Stroke. 2015;46:91-7.

31. Wang A, Liu X, Chen G, Hao H, Wang Y, Wang Y. Association between carotid plaque and cognitive impairment in Chinese stroke population: the SOS-Stroke Study. Sci Rep. 2017;7:3066.

32. Yokota K, Fukushima M, Takahashi Y, Igaki N, Seino S. Insulin secretion and computed tomography values of the pancreas in the early stage of the development of diabetes. J Diabetes Investig. 2012;20:3:371-6.

33. Wong VW, Wong GL, Yeung DK, Abrigo JM, Kong AP, Chan RS, et al. Fatty pancreas, insulin resistance, and $\beta$-cell function: a population study using fat-water magnetic resonance imaging. Am J Gastroenterol. 2014;109:589-97.

34. Kitada M, Zhang Z, Mima A, King GL. Molecular mechanisms of diabetic vascular complications. J Diabetes Investig. 2010;1:77-89. 